\title{
A Three-phase Hybrid Power Flow Algorithm for Meshed Distribution System with Transformer Branches and PV Nodes
}

\author{
Hongwei Li ${ }^{\dagger}$, Huabing Wu*, Biyu Jiang*, Anan Zhang* and Wei Fang*
}

\begin{abstract}
Aiming at analyzing the power flow of the distribution systems with distribution transformer (DT) branches and PV nodes, a hybrid three-phase power flow methodology is presented in this paper. The incidence formulas among node voltages, loop currents and node current injections have been developed based on node-branch incidence matrix of the distribution network. The method can solve the power flow directly and has higher efficiency. Moreover, the paper provides a modified method to model DT branches by considering winding connections, phase shifting and off-nominal tap ratio, and then DT branches could be seen like one transmission line with the proposed power flow method. To deal with the PV nodes, an improved approach to calculate reactive power increment at each PV node was deduced based on the assumption that the positive-sequence voltage magnitude of $\mathrm{PV}$ node is fixed at a given value. Then during calculating the power flow at each iteration, it only needs to update current injection at each PV node with the proposed algorithm. The process is very simple and clear. The results of IEEE 4 nodes and the modified IEEE 34 nodes test feeders verified the correctness and efficiency of the proposed hybrid power flow algorithm.
\end{abstract}

Keywords: Distribution transformer, Meshed distribution system, Node-branch incidence matrix, Power flow, PV nodes

\section{Introduction}

Power flow method is a fundamental tool for distribution management system, so a robust and efficient power flow solution method must be able to model the special features of distribution systems in sufficient detail. During the past decade, several power flow algorithms have been developed [1-11]. The most popular estimation algorithm is the forward/backward sweep based method for its fully utilizing the distribution network structure characteristics, simple programming, no large matrix calculation and fast computing speed [6-11].

For meshed network, the most commonly adopted solution is the compensation current method [6-7]. It uses a reduced Thévenin impedance matrix and a vector of known terms that are the open circuit voltages between the cut nodes. The method in [8] is a direct extension of the compensation-based power flow method proposed earlier in [6-7], and it is capable of handling three-phase weakly meshed distribution network, dispersed generation (PV nodes) and unbalanced and distributed loads. The good results have been achieved with this method, but it has more iterations and becomes less efficient when node voltages are lower. A direct approach for unbalanced three-phase distribution power flow solutions is proposed

$\dagger$ Corresponding Author: School of Electrical and Electronic Engineering, Southwest Petroleum University, Chengdu, China. (lhwmail@126.com)

* School of Electrical and Electronic Engineering, Southwes Petroleum University, Chengdu, China. (\{363116099, 461802287\} @qq.com, ananzhang@swpu.edu.cn, villa1006@163.com)

Received: December 2, 2013; Accepted: October 5, 2015 in [9], in which two developed matrices, the bus-injection to branch-current matrix and the branch-current to busvoltage matrix, are used to solve power flow problem directly. The proposed method is robust and time-efficient, but the universal forms of two matrices are not proved through theoretical deduction. Reference [10] presents a novel forward/backward sweep algorithm for meshed network which is firmly based on loop analysis theorem. The method gives a concise formula to formulate the relationship between branch flow and node injection flow. The convergence of this novel algorithm is improved dramatically for meshed network. Reference [11] developed a methodology to analyze the radial or weakly meshed distribution systems, in which loads are simulated by means of impedances. The applications show the efficiency of the methodology in solving distribution networks with many meshes and PV nodes.

Detailed models for three-phase distribution transformers (DTs) are critical for distribution system analysis. The most common DT models are based on nodal admittance matrices [12 18]. But for the forward/backward sweep based methods, the nodal admittance matrices based models are proved to be difficult to be used and claimed to be unsatisfactory due to relatively slow convergence problems [17]. It may also cause singularity in the connection matrix which appears on some special connection types such as delta-grounded wye or grounded wye-delta transformers. Moreover, it also need considers the different neutral grounding modes and the difference between zero sequence impedance and positive sequence 
impedance

The future distribution networks will consist of a considerable proportion distributed generators (DGs) based on renewable energy sources. The emergence of DGs into the distribution systems has great influence on the voltage quality, power flow and loss etc. [19-20]. So far, many related researches have been done and many algorithms have been proposed. Reference [21] introduces an improved Newton-Raphson algorithm with lower initial values requirement. But the method is much complicated to deal with PV nodes and the three-phase power. Besides, it needs to calculate the Jacobian matrix and the matrix may be illconditioned for the high $\mathrm{R} / \mathrm{X}$ ratios in distribution system. The most commonly adopted method in the solution scheme is also the forward/backward sweep techniques [6$8,11,22-23]$. In $[6-8,11]$, the method based on current compensation for multi-port systems is applied to solve radial and weakly meshed systems with PV nodes and have good efficiency. But the converged iteration increases significantly, and sometimes the results do not converge when network become more meshed and has more PV nodes. In [22], based on the models of DGs (wind turbines, photovoltaic system, fuel cell, CHP etc.) for power flow calculation, an improved three-phase back/forward sweep power flow calculation for the weakly meshed distribution network with the distributed generation was presented. Reference [23] proposed a direct unbalanced three-phase power flow algorithm and a new approach handling PV nodes based on the assumption that the positive-sequence voltage magnitudes of PV nodes are invariable. The efficiency and feasibility of the two algorithms has been proved, but they didn't consider the transformer branches in the meshed network.

In a word, the power flow algorithms need to be further studied considering transformer branches and the high penetration of DGs into the power distribution system. This paper develops a three-phase power flow solution method for real-time analysis of meshed distribution systems with DT branches and PV nodes. The focus of this paper is on the improved node-branch incidence matrix based power flow algorithm and several modeling issues related to distribution system including DT branches and DGs (PV nodes). The test results show the efficiency of the proposed method in solving distribution networks in complex situations. The modeling detail and convergence performance of the algorithm make it suitable for real-time applications in distribution automation systems.

\section{Node-branch Incidence Matrix Based Power Flow Method}

For a weakly meshed distribution network with $N+1$ nodes and $m$ link branches (loops), there are $N$ independent nodes when the power supply is referred as reference node (the first node). The number of the total braches $b$ equals $N+m$. The reduced node-branch incidence matrix $\mathbf{A}$ can be used to describe the network. The element in $\mathbf{A}$ is [24]:

$$
a_{i j}=\left\{\begin{array}{c}
-1 \text { Node } i \text { is the starting point of branch } j \\
1 \text { Node } i \text { is the ending point of branch } j \\
0 \text { Node } i \text { is neither the starting point nor } \\
\text { the ending point of branch } j
\end{array}\right.
$$

Designating one tree in the weakly meshed network and numbering the tree branches in the front and the link branches in the back, then $\mathbf{A}$ can be expressed as,

$$
\mathbf{A}=\left[\mathbf{A}_{\mathrm{t}}, \mathbf{A}_{l}\right]
$$

The order of invertible $\mathbf{A}_{\mathrm{t}}$ is $N \times N$ and the order of $\mathbf{A}_{l}$ is $N \times l$.

Excluding the node injection branches from the network and assuming that the node injection current is positive if it flows out of the node and node injection current is negative if it flows into the node. Let $\boldsymbol{I}_{\mathrm{b}}=\left[\begin{array}{c}\boldsymbol{I}_{\mathrm{bt}} \\ \boldsymbol{I}_{\mathrm{b} l}\end{array}\right]$ be the branch current vector of $b \times 1$ and $\boldsymbol{I}_{\mathrm{g}}$ be the node injection current vector of $N \times 1$, there exists,

$$
\boldsymbol{I}_{\mathrm{g}}=\mathbf{A} \boldsymbol{I}_{\mathrm{b}}=\left[\mathbf{A}_{\mathrm{t}}, \mathbf{A}_{l}\right]\left[\begin{array}{c}
\boldsymbol{I}_{\mathrm{bt}} \\
\boldsymbol{I}_{\mathrm{b} l}
\end{array}\right]=\mathbf{A}_{\mathrm{t}} \boldsymbol{I}_{\mathrm{bt}}+\mathbf{A}_{l} \boldsymbol{I}_{\mathrm{b} l}
$$

where, $\boldsymbol{I}_{\mathrm{bt}}$ is the tree branch current vector of $N \times 1, \quad \boldsymbol{I}_{\mathrm{b} l}$ is the loop current (link branch current) vector of $l \times 1$.

Then $\boldsymbol{I}_{\mathrm{bt}}$ can be obtained with,

$$
\boldsymbol{I}_{\mathrm{bt}}=\mathbf{A}_{\mathrm{t}}^{-1} \boldsymbol{I}_{\mathrm{g}}-\mathbf{A}_{\mathrm{t}}^{-1} \mathbf{A}_{l} \boldsymbol{I}_{\mathrm{b} l}=\mathbf{T}_{\mathrm{t}}^{\mathrm{T}} \boldsymbol{I}_{\mathrm{g}}+\mathbf{B}_{\mathrm{t}}^{\mathrm{T}} \boldsymbol{I}_{\mathrm{b} l}
$$

where, Superscript "T" indicates matrix transpose. $\mathbf{B}_{\mathrm{t}}\left(\mathbf{B}_{\mathrm{t}}^{\mathrm{T}}=-\mathbf{A}_{\mathrm{t}}^{-1} \mathbf{A}_{l}=-\mathbf{T}_{\mathrm{t}}^{\mathrm{T}} \mathbf{A}_{l}\right)$ is the loop matrix of $l \times N$ and $\mathbf{T}_{\mathrm{t}}\left(\mathbf{T}_{\mathrm{t}}^{\mathrm{T}}=\mathbf{A}_{\mathrm{t}}^{-1}\right)$ is the path matrix of $N \times N$ corresponding to tree branches [10]. Define $\boldsymbol{f}_{1}=\boldsymbol{T}_{\mathrm{t}}^{\mathrm{T}} \boldsymbol{I}_{\mathrm{g}}$, the contribution of node current injection $\boldsymbol{I}_{\mathrm{g}}$, and $\boldsymbol{f}_{2}=\boldsymbol{B}_{\mathrm{t}}^{\mathrm{T}} \boldsymbol{I}_{l}$, the contribution of loop current $\boldsymbol{I}_{\mathrm{b} l}$.

Based on Kirchhoff's voltage law and Ohm law,

$$
\boldsymbol{B} \boldsymbol{U}_{\mathrm{b}}=\left[\begin{array}{ll}
\boldsymbol{B}_{\mathrm{t}} & \boldsymbol{E}_{l}
\end{array}\right]\left[\begin{array}{l}
\boldsymbol{U}_{\mathrm{bt}} \\
\boldsymbol{U}_{\mathrm{b} l}
\end{array}\right]=\left[\begin{array}{ll}
\boldsymbol{B}_{\mathrm{t}} & \boldsymbol{E}_{l}
\end{array}\right]\left[\begin{array}{cc}
\boldsymbol{Z}_{\mathrm{bt}} & \mathbf{0} \\
\mathbf{0} & \boldsymbol{Z}_{\mathrm{b} l}
\end{array}\right]\left[\begin{array}{l}
\boldsymbol{I}_{\mathrm{bt}} \\
\boldsymbol{I}_{\mathrm{b} l}
\end{array}\right]=0
$$

where, $\boldsymbol{E}_{l}$ is unit matrix of $l \times l, \boldsymbol{U}_{\mathrm{bt}}$ is the tree branch corresponding voltage vector of $N \times 1, \quad \boldsymbol{U}_{\mathrm{b} l}$ is the link branch corresponding voltage vector of $l \times 1, Z_{\mathrm{bt}}$ is the tree branch corresponding impedance diagonal matrix of $N \times N, \boldsymbol{Z}_{\mathrm{b} l}$ is the link branch corresponding impedance diagonal matrix of $l \times l$.

Expanding (4) and rearranging it gives,

$$
\boldsymbol{B}_{\mathrm{t}} \boldsymbol{Z}_{\mathrm{bt}} \boldsymbol{I}_{\mathrm{bt}}+\boldsymbol{Z}_{\mathrm{bl}} \boldsymbol{I}_{\mathrm{b} l}=0
$$


Substituting (3) into (5), $\boldsymbol{I}_{\mathrm{b} l}$ can be derived as,

$$
\boldsymbol{I}_{\mathrm{b} l}=-\boldsymbol{Y}_{l} \boldsymbol{B}_{\mathrm{t}} \boldsymbol{Z}_{\mathrm{bt}} \boldsymbol{T}_{\mathrm{t}}^{\mathrm{T}} \boldsymbol{I}_{g}=\boldsymbol{Y}_{l} \Delta \boldsymbol{U}_{l}
$$

where, $\quad \Delta \boldsymbol{U}_{l}=-\boldsymbol{B}_{\mathrm{t}} \boldsymbol{Z}_{\mathrm{bt}} \boldsymbol{T}_{\mathrm{t}}^{\mathrm{T}} \boldsymbol{I}_{g}, \boldsymbol{Z}_{l}=\boldsymbol{B}_{\mathrm{t}} \boldsymbol{Z}_{\mathrm{bt}} \boldsymbol{B}_{\mathrm{t}}^{\mathrm{T}}+\boldsymbol{Z}_{\mathrm{b} l}$ is the loop impedance matrix. $\boldsymbol{Y}_{l}$ Is the inverse matrix of $\boldsymbol{Z}_{l}$.

Let the nodes at the both ends of link branch in loop $h$ be $i$ and $j$, then the contribution of $\dot{I}_{l h}$ in loop $h$ to the tree branch currents is,

$$
\boldsymbol{f}_{2 h}=\boldsymbol{B}_{\mathrm{th}}^{\mathrm{T}} \boldsymbol{I}_{l h}=\left[\boldsymbol{T}_{\mathrm{t} i}-\boldsymbol{T}_{\mathrm{t} j}\right]^{\mathrm{T}} \dot{I}_{l h}
$$

where, $\dot{I}_{l h}$ is assumed to flow from node $i$ to node $j$, $\boldsymbol{T}_{\mathrm{t} i}$ and $\boldsymbol{T}_{\mathrm{t} j}$ are the node $i$ and $j$ corresponding row vectors(path vectors)in $\boldsymbol{T}_{\mathrm{t}}, \boldsymbol{B}_{\mathrm{t} h}=\left[\boldsymbol{T}_{\mathrm{t} i}-\boldsymbol{T}_{\mathrm{t} j}\right]$ is the loop $h$ corresponding row vector in $\boldsymbol{B}_{\mathrm{t}}$.

The contributions of other loop currents to the tree branch current can be also calculated with (7), so another form of $\boldsymbol{f}_{2}$ can be expressed as,

$$
\boldsymbol{f}_{2}=\sum_{h=1}^{m} \boldsymbol{f}_{2 h}
$$

The voltage difference $\Delta \boldsymbol{U}_{\mathrm{t} i}$ between power supply and any other node $i$ is equal to the sum of tree branch voltage along the path of node $i$, that is:

$$
\Delta \boldsymbol{U}_{\mathrm{t} i}=\boldsymbol{U}_{0}-\boldsymbol{U}_{\mathrm{n} i}=\boldsymbol{T}_{\mathrm{t} i} \boldsymbol{Z}_{\mathrm{bt}} \boldsymbol{I}_{\mathrm{bt}} \quad(i=1,2, \cdots, N)
$$

where $\boldsymbol{U}_{0}$ is the power supply voltage and $\boldsymbol{U}_{\mathrm{n}}$ is the node voltage vector of $N \times 1$.

The power flow calculation can be realized by solving (9) iteratively. The improved algorithm can be described as following (set $k$ as iteration count):

Step 1: Calculate nodal current: the node $i$ injection current $\dot{I}_{g i}^{k}$ is calculated by,

$$
\dot{I}_{\mathrm{g} i}^{k}=\left(S_{i} / \dot{U}_{\mathrm{n} i}^{(k-1)}\right)^{*}-Y_{i} \dot{U}_{\mathrm{n} i}^{(k-1)} \quad(i=1,2, \cdots, n)
$$

where, $\dot{U}_{\mathrm{n} i}^{(k-1)}$ is the voltage, $S_{p i}$ is the power injection and $Y_{i}$ is the sum of all the shunt elements at node $i$.

Step 2: Calculate $\boldsymbol{f}_{1}^{k}=\boldsymbol{T}_{\mathrm{t}}^{\mathrm{T}} \boldsymbol{I}_{\mathrm{g}}^{k}$, which is equivalent to break the links and do a backward sweep calculation for the radial network.

Step 3: Calculate $\boldsymbol{f}_{2}^{k}$ with (6), (7) and (8), then calculate branch current $\boldsymbol{I}_{\mathrm{bt}}^{k}$ with (3).

Step 4: Calculate $\Delta \boldsymbol{U}_{\mathrm{n} i}^{k}$ with (9), and the nodal voltage is updated with $\boldsymbol{U}_{\mathrm{n} i}^{k}=\boldsymbol{U}_{0}-\Delta \boldsymbol{U}_{\mathrm{n} i}^{k}$;

Steps 1-4 are repeated until convergence is achieved (here, when the maximum voltage mismatch between $\boldsymbol{U}_{\mathrm{n}}^{k}$ and $\boldsymbol{U}_{\mathrm{n}}^{k-1}$ is small enough).

The above algorithm is also suitable for the three-phase unbalanced system and it only need to update the elements in each vector (matrix) with three-phase system parameters.

\section{Handling of DT Branch in Power Flow Algorithm}

\subsection{Modeling of distribution transformer}

The exact DT models of the various connections must satisfy Kirchhoff's voltage and current laws (KVL and $\mathrm{KCL}$ ) and the ideal relationship between the voltages and currents on the two sides of the DT windings. The models must also represent any phase shift because of the particular connection. DT branch sketch is shown in Fig. 1. The core loss of DT could be evaluated by the shunt core loss functions on each phase of the secondary terminal [12] and will not be considered in the models.

Let DT line-to-ground voltage be $\boldsymbol{U}_{\mathrm{PG}}=\left[\begin{array}{lll}\dot{U}_{\mathrm{AG}} & \dot{U}_{\mathrm{BG}} & \dot{U}_{\mathrm{CG}}\end{array}\right]^{\mathrm{T}}$ and $\boldsymbol{U}_{\mathrm{sg}}=\left[\begin{array}{lll}\dot{U}_{\mathrm{ag}} & \dot{U}_{\mathrm{bg}} & \dot{U}_{\mathrm{cg}}\end{array}\right]^{\mathrm{T}}$, DT line-to-neutral voltage be $\boldsymbol{U}_{\mathrm{sn}}=\left[\begin{array}{lll}\dot{U}_{\mathrm{an}} & \dot{U}_{\mathrm{bn}} & \dot{U}_{\mathrm{cn}}\end{array}\right]^{\mathrm{T}}$, DT line currents be $\boldsymbol{I}_{p}=\left[\begin{array}{lll}\dot{I}_{\mathrm{A}} & \dot{I}_{\mathrm{B}} & \dot{I}_{\mathrm{C}}\end{array}\right]^{\mathrm{T}}$ and $\boldsymbol{I}_{\mathrm{s}}=\left[\begin{array}{lll}\dot{I}_{\mathrm{a}} & \dot{I}_{\mathrm{b}} & \dot{I}_{\mathrm{c}}\end{array}\right]^{\mathrm{T}}$. Subscript " $p$ " and " $s$ " indicate primary side and secondary side respectively.

There exist the following equations,

$$
\begin{aligned}
\boldsymbol{I}_{\mathrm{p}} & =\boldsymbol{H}_{11} \boldsymbol{U}_{\mathrm{pG}}+\boldsymbol{H}_{12} \boldsymbol{I}_{\mathrm{s}} \\
\boldsymbol{U}_{\mathrm{s}} & =\boldsymbol{H}_{21} \boldsymbol{U}_{\mathrm{pG}}-\boldsymbol{H}_{22} \boldsymbol{I}_{\mathrm{s}}
\end{aligned}
$$

When solving the power flow, $\boldsymbol{U}_{\mathrm{s}}$ chooses $\boldsymbol{U}_{\mathrm{sn}}$ for ungrounded wye-connection and delta-connection and chooses $\boldsymbol{U}_{\mathrm{sg}}$ for wye-grounded connection.

For delta-connection in secondary side, the equivalent line-to-neutral voltages is determined by,

$$
\boldsymbol{U}_{\mathrm{sn}}=\boldsymbol{A} \boldsymbol{Z}_{\mathrm{T} 012} \frac{1}{\sqrt{3}}\left[\begin{array}{ccc}
0 & 0 & 0 \\
0 & 1 \angle-30^{\circ} & 0 \\
0 & 0 & 1 \angle 30^{\circ}
\end{array}\right] \boldsymbol{A}^{-1} \boldsymbol{U}_{\mathrm{sL}}=\boldsymbol{K} \boldsymbol{U}_{\mathrm{sL}}
$$

where, $\boldsymbol{A}=\left[\begin{array}{llllll}1 & 1 & 1 ; 1 & \mathrm{a}^{2} & \text { a; } & \mathrm{a} \mathrm{a}^{2}\end{array}\right]$

$\left(\mathrm{a}=e^{\mathrm{j} \frac{2 \pi}{3}}\right), \quad \boldsymbol{K}=[10-1 ;-1110 ; 0-11] / 3$,

$\boldsymbol{U}_{\mathrm{sL}}=\left[\begin{array}{lll}\dot{U}_{\mathrm{ab}} & \dot{U}_{\mathrm{bc}} & \dot{U}_{\mathrm{ca}}\end{array}\right]^{\mathrm{T}}$.

Let $z_{\mathrm{t} 1}$ be DT positive-sequence impedance and $z_{\mathrm{t} 0}$ be DT zero-sequence impedance, then let $\boldsymbol{Z}_{\mathrm{T} 012}=$ $\operatorname{diag}\left[\begin{array}{lll}z_{\mathrm{t} 0} & z_{\mathrm{t} 1} & z_{\mathrm{t} 1}\end{array}\right]$ be three-sequence impedance matrix. So the three-phase DT impedance matrix is,

$$
\boldsymbol{Z}_{\text {phase }}=\boldsymbol{A} \boldsymbol{Z}_{\mathrm{T} 012} \boldsymbol{A}^{-1}=\frac{1}{3}\left[\begin{array}{ccc}
z_{\mathrm{t} 0}+2 z_{\mathrm{t} 1} & z_{\mathrm{t} 0}-z_{\mathrm{t} 1} & z_{\mathrm{t}}-z_{\mathrm{t} 1} \\
z_{\mathrm{t} 0}-z_{\mathrm{t} 1} & z_{\mathrm{t} 0}+2 z_{\mathrm{t} 1} & z_{\mathrm{t} 0}-z_{\mathrm{t} 1} \\
z_{\mathrm{t} 0}-z_{\mathrm{t} 1} & z_{\mathrm{t} 0}-z_{\mathrm{t} 1} & z_{\mathrm{t} 0}+2 z_{\mathrm{t} 1}
\end{array}\right]
$$

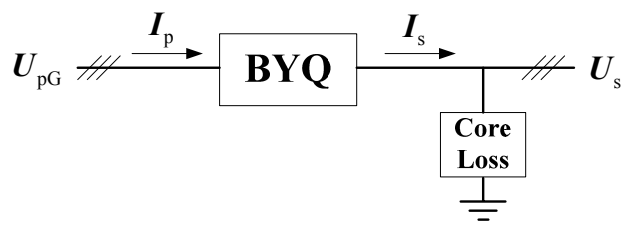

Fig. 1. The sketch of transformer branch 
Referring to the modeling process in $[18,26]$ and further considering DT impedance asymmetry and neutral point grounded impedance, the exact DT models and coefficient matrices in (11) were re-derived (derivation process is omitted). The coefficient matrices for all kind of DT connections were shown in Table 1.

Table 1. The coefficient matrices of transformer models

\begin{tabular}{c|c|c|c|c}
\hline Connection & $\boldsymbol{H}_{11}$ & $\boldsymbol{H}_{12}$ & $\boldsymbol{H}_{21}$ & $\boldsymbol{H}_{22}$ \\
\hline $\mathrm{Y}_{\mathrm{n}}-\mathrm{y}_{\mathrm{n}}$ & 0 & $1 / n_{\mathrm{T}}$ & $1 / n_{\mathrm{T}}$ & $\beta^{2} \boldsymbol{Z}_{\text {phase }}$ \\
\hline $\mathrm{Y}_{-} \mathrm{y}_{\mathrm{n}}$ & 0 & $\boldsymbol{W} / n_{\mathrm{T}}$ & $\boldsymbol{W} / n_{\mathrm{T}}$ & $\beta^{2} \boldsymbol{Z}_{\text {phase }}$ \\
\hline $\mathrm{D}^{-} \mathrm{y}_{\mathrm{n} 11}$ & 0 & $\sqrt{3} \boldsymbol{K} / n_{\mathrm{T}}$ & $\sqrt{3} \boldsymbol{K}^{\mathrm{T}} / n_{\mathrm{T}}$ & $\beta^{2} \boldsymbol{Z}_{\text {phase }}$ \\
\hline $\mathrm{Y}_{\mathrm{n}}-\mathrm{d}_{11}$ & $\boldsymbol{M} /\left(3 \alpha^{2} z_{\mathrm{t} 0}\right)$ & $\sqrt{3} \boldsymbol{K} / n_{\mathrm{T}}$ & $\sqrt{3} \boldsymbol{K}^{\mathrm{T}} / n_{\mathrm{T}}$ & $\beta^{2} z_{\mathrm{t} 1} \boldsymbol{W} / 3$ \\
\hline $\mathrm{Y}_{11}$ & 0 & $\sqrt{3} \boldsymbol{K} / n_{\mathrm{T}}$ & $\sqrt{3} \boldsymbol{K}^{\mathrm{T}} / n_{\mathrm{T}}$ & $\beta^{2} z_{\mathrm{t}} \boldsymbol{W} / 3$ \\
\hline $\mathrm{D}-\mathrm{d}$ & 0 & $1 / n_{\mathrm{T}}$ & $\boldsymbol{W} / n_{\mathrm{T}}$ & $\beta^{2} z_{\mathrm{t}} \boldsymbol{W} / 3$ \\
\hline $\mathrm{D}-\mathrm{y}_{11}$ & 0 & $\sqrt{3} \boldsymbol{K} / n_{\mathrm{T}}$ & $\sqrt{3} \boldsymbol{K}^{\mathrm{T}} / n_{\mathrm{T}}$ & $\beta^{2} z_{\mathrm{t} 1}$ \\
\hline $\mathrm{Y}_{\mathrm{n}}-\mathrm{y}$ & 0 & $1 / n_{\mathrm{T}}$ & $1 / n_{\mathrm{T}}$ & $\beta^{2} z_{\mathrm{t} 1}$ \\
\hline $\mathrm{Y}-\mathrm{y}$ & 0 & $1 / n_{\mathrm{T}}$ & $1 / n_{\mathrm{T}}$ & $\beta^{2} z_{\mathrm{t} 1}$ \\
\hline & &
\end{tabular}

where, $n_{\mathrm{T}}=\alpha / \beta$ is the off-nominal DT tap ratio ( $\alpha$ and $\beta$ are the taps on the two sides respectively), $\boldsymbol{W}=[2-1-1 ;-12-1 ;-1-12] / 3$ and $\boldsymbol{M}$ is all-1 matrix of $3 \times 3$. It needs to note that $z_{\mathrm{t} 0}$ should be replaced with $z_{\mathrm{t} 0}+3 z_{\mathrm{tg}}$ for $\mathrm{Y}-\mathrm{y}_{\mathrm{n}}, \mathrm{D}-\mathrm{y}_{\mathrm{n} 11}$ and $\mathrm{Y}_{\mathrm{n}}-\mathrm{d}_{11}$ if there is a grounded impedance $z_{\mathrm{tg}}$ at neutral point.

\subsection{Handling of DT branch}

In Table 1, except $\mathrm{Y}_{\mathrm{n}}-\mathrm{d}_{11}$, all other connections have $\boldsymbol{H}_{11}=0$. Even for $\mathrm{Y}_{\mathrm{n}}-\mathrm{d}_{11}, \boldsymbol{H}_{11} \boldsymbol{U}_{\mathrm{p}}$ can also be removed from (11a) because $\boldsymbol{H}_{11} \boldsymbol{U}_{\mathrm{p}}$ is generally much smaller than $\boldsymbol{H}_{12} \boldsymbol{I}_{\mathrm{s}}$. So (11a) can be simplified as:

$$
\boldsymbol{I}_{\mathrm{p}}=\boldsymbol{H}_{12} \boldsymbol{I}_{\mathrm{s}}=\boldsymbol{K}_{\mathrm{I}} \boldsymbol{I}_{\mathrm{s}}
$$

Define $\boldsymbol{U}_{\mathrm{s}}^{\prime}=\boldsymbol{H}_{21} \boldsymbol{U}_{\mathrm{p}}=\boldsymbol{K}_{\mathrm{V}} \boldsymbol{U}_{\mathrm{p}} \quad$ and $\quad \boldsymbol{Z}_{\mathrm{T}}=\boldsymbol{H}_{22} \quad, \quad$ and substituting them into (11b),

$$
\boldsymbol{U}_{\mathrm{s}}=\boldsymbol{K}_{\mathrm{V}} \boldsymbol{U}_{\mathrm{p}}-\boldsymbol{Z}_{\mathrm{T}} \boldsymbol{I}_{\mathrm{s}}=\boldsymbol{U}_{\mathrm{s}}^{\prime}-\boldsymbol{Z}_{\mathrm{T}} \boldsymbol{I}_{\mathrm{s}}
$$

where, $\boldsymbol{K}_{\mathrm{I}}=\boldsymbol{H}_{12}$ is the voltage transformation matrix and $\boldsymbol{K}_{\mathrm{V}}=\boldsymbol{H}_{21}$ is the current transformation matrix. Note the diagonal entries in the matrices, $\boldsymbol{K}_{\mathrm{I}}$ is not equal to $\boldsymbol{K}_{\mathrm{V}}$ from the derivation results earlier.

Hence, based on (15), the DT branch can be described with an equivalent model shown in Fig. 2.

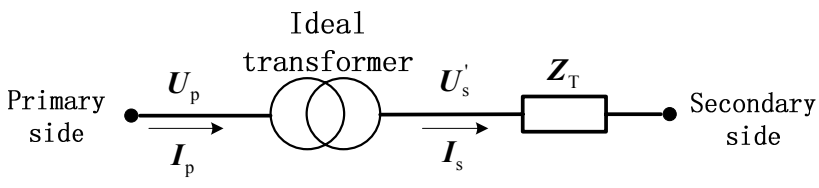

Fig. 2. The equivalent model of DT branch

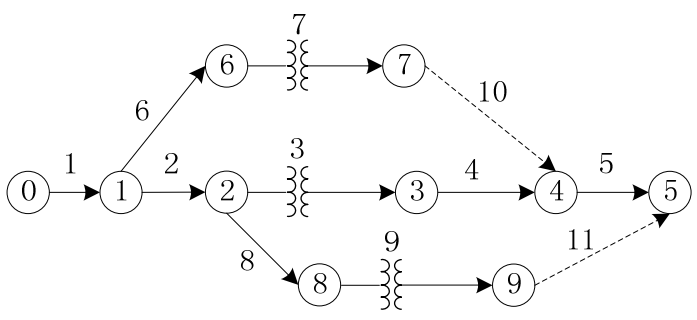

Fig. 3. A simple network with transformer branch

\subsection{Improved power flow algorithm with DT}

For the network with the DT, $\boldsymbol{I}_{\mathrm{bt}}$ and $\boldsymbol{U}_{\mathrm{n}}$ can't be directly calculated with proposed algorithm in section 2 for current and voltage transformations. So based on the network shown in Fig.3 as follows, the improvement to calculate $\boldsymbol{I}_{\mathrm{b}}$ and $\boldsymbol{U}_{\mathrm{n}}$ were analyzed and re-derived again.

There are three DT branches and two loops in Fig.3, in which $\boldsymbol{K}_{\mathrm{V} 3}, \boldsymbol{K}_{\mathrm{V} 7}, \boldsymbol{K}_{\mathrm{V} 9}$ and $\boldsymbol{K}_{\mathrm{I} 3}, \boldsymbol{K}_{\mathrm{I} 7}, \boldsymbol{K}_{\mathrm{I} 9}$ can be calculated with the DT coefficient matrices.

\subsubsection{Calculation of $f_{1}$}

Let $\boldsymbol{I}_{\mathrm{s}}$ be the DT branch current, and the second branch current, $\boldsymbol{I}_{\mathrm{b} 2}$, in Fig. 3 can be calculated by ( $\boldsymbol{E}$ is unit matrix of $3 \times 3$ and 0 is all- 0 matrix of $3 \times 3$ ):

$$
\begin{aligned}
& \boldsymbol{I}_{\mathrm{b} 2}=\boldsymbol{I}_{\mathrm{g} 2}+\boldsymbol{K}_{\mathrm{l} 3}\left(\boldsymbol{I}_{\mathrm{g} 3}+\boldsymbol{I}_{\mathrm{g} 4}\right)+\boldsymbol{I}_{\mathrm{g} 7}+\boldsymbol{K}_{\mathrm{I} 8} \boldsymbol{I}_{\mathrm{g} 8} \\
& =\left[\begin{array}{llllllll}
\boldsymbol{0} & \boldsymbol{E} & \boldsymbol{K}_{\mathrm{I} 3} & \boldsymbol{K}_{\mathrm{I} 3} & \boldsymbol{O} & \boldsymbol{O} & \boldsymbol{E} & \boldsymbol{K}_{\mathrm{I} 8}
\end{array}\right] \boldsymbol{I}_{\mathrm{g}}
\end{aligned}
$$

Similarly, other branch currents can be obtained too. So $f_{1}$ can be expressed in general form as,

$$
\boldsymbol{f}_{1}=\boldsymbol{T}_{\mathrm{t} K_{\mathrm{I}}}^{\mathrm{T}} \boldsymbol{I}_{\mathrm{g}}
$$

Substituting all corresponding $\boldsymbol{K}_{\mathrm{I}}$ with $\boldsymbol{K}_{\mathrm{V}}$ in $\boldsymbol{T}_{\mathrm{t} K_{\mathrm{I}}}$ can get $\boldsymbol{T}_{\mathrm{t} K_{\mathrm{V}}} . \boldsymbol{T}_{\mathrm{t} i K_{\mathrm{V}}}$ is the node $i$ corresponding row vector (path) in $\boldsymbol{T}_{\mathrm{t} K_{\mathrm{V}}}$. Supposing that $\boldsymbol{f}_{2}$ is known, $\boldsymbol{I}_{\mathrm{bt}}$ can be calculated by (2). The voltage at node $i$ can be obtained by,

$$
\boldsymbol{U}_{\mathrm{n} i}=\boldsymbol{K}_{0 i} \boldsymbol{U}_{0}-\Delta \boldsymbol{U}_{\mathrm{ti}}=\boldsymbol{K}_{0 i} \boldsymbol{U}_{0}-\boldsymbol{T}_{\mathrm{t} i K_{\mathrm{V}}} \boldsymbol{Z}_{\mathrm{bt}} \boldsymbol{I}_{\mathrm{bt}}(i=1,2, \cdots, N)
$$

where, $\boldsymbol{K}_{0 i}$ is the first entry of $\boldsymbol{T}_{\mathrm{t} i K_{\mathrm{V}}}$.

Appendix A describes the generation of $\boldsymbol{T}_{\mathrm{t} K_{\mathrm{I}}}$ and the derivation of (17) in detail.

\subsubsection{Calculation of $\boldsymbol{f}_{2}$}

To calculate $\boldsymbol{f}_{2}$ needs to calculate $\boldsymbol{I}_{l}$ firstly. But from (6), to calculate $\boldsymbol{I}_{l}$ must know $\Delta \boldsymbol{U}_{l}$ and $\boldsymbol{Y}_{l}$. In fact, $\Delta \boldsymbol{U}_{l}$ is the voltage difference vector of $3 m \times 1$ between the both ends of link branch when breaking link branches and only considering the contribution of $\boldsymbol{f}_{1}$. Let the ends of link branch in loop $h$ be $i$ and $j$, then $\boldsymbol{U}_{\mathrm{n} i}$ and $\boldsymbol{U}_{\mathrm{n} j}$ can be calculated by (17) (here, $\boldsymbol{I}_{\mathrm{bt}}=\boldsymbol{f}_{1}$ not including $\boldsymbol{f}_{2}$ ). 
So $\Delta \boldsymbol{U}_{l h}$ is,

$$
\Delta \boldsymbol{U}_{l h}=\boldsymbol{U}_{\mathrm{n} i}-\boldsymbol{U}_{\mathrm{n} j}
$$

To calculate $\boldsymbol{Y}_{l}$ should calculate $\boldsymbol{Z}_{l}$ first. In Fig. 1, the following equation is true,

$$
\begin{aligned}
\boldsymbol{U}_{\mathrm{s}^{\prime}} & =\boldsymbol{K}_{\mathrm{V}} \boldsymbol{U}_{\mathrm{p}}=\boldsymbol{K}_{\mathrm{V}}\left(\boldsymbol{U}_{\mathrm{p} 0}-\boldsymbol{Z}_{\mathrm{p}} \boldsymbol{I}_{\mathrm{p}}\right) \\
& =\boldsymbol{K}_{\mathrm{V}} \boldsymbol{U}_{\mathrm{p} 0}-\boldsymbol{K}_{\mathrm{V}} \boldsymbol{Z}_{\mathrm{p}} \boldsymbol{K}_{\mathrm{I}} \boldsymbol{I}_{\mathrm{s}}
\end{aligned}
$$

where, $\boldsymbol{U}_{\mathrm{p} 0}$ is the power supply voltage and, referring to DT primary side, $\boldsymbol{Z}_{\mathrm{p}}$ can be seen as the internal resistance of the power supply.

It can be seen from (19) that $\boldsymbol{Z}_{\mathrm{p}}$ became $\boldsymbol{K}_{\mathrm{V}} \boldsymbol{Z}_{\mathrm{p}} \boldsymbol{K}_{\mathrm{I}}$ when it is referred to DT secondary side. There are two loops in Fig. 3, and the self and mutual impedances are ( $\boldsymbol{Z}_{l}$ is referred to DT secondary side because $\Delta \boldsymbol{U}_{l}$ is the voltage vector in DT secondary side):

$$
\begin{aligned}
& \boldsymbol{Z}_{l_{-} 11}=\boldsymbol{K}_{\mathrm{v} 3} \boldsymbol{Z}_{\mathrm{b} 2} \boldsymbol{K}_{\mathrm{1} 3}+\boldsymbol{Z}_{\mathrm{T} 3}+\boldsymbol{Z}_{\mathrm{b} 4}+\boldsymbol{K}_{\mathrm{v} 7} \boldsymbol{Z}_{\mathrm{b} 6} \boldsymbol{K}_{\mathrm{I} 7}+\boldsymbol{Z}_{\mathrm{T} 7}+\boldsymbol{Z}_{\mathrm{b} 10} \\
& \boldsymbol{Z}_{l_{-} 22}=\boldsymbol{Z}_{\mathrm{T} 3}+\boldsymbol{Z}_{\mathrm{b} 4}+\boldsymbol{Z}_{\mathrm{b} 5}+\boldsymbol{K}_{\mathrm{v} 9} \boldsymbol{Z}_{\mathrm{b} 8} \boldsymbol{K}_{\mathrm{I} 9}+\boldsymbol{Z}_{\mathrm{T} 11}+\boldsymbol{Z}_{\mathrm{b} 11} \\
& \boldsymbol{Z}_{l_{-} 12}=\boldsymbol{Z}_{l_{-} 21}=\boldsymbol{Z}_{\mathrm{T} 3}+\boldsymbol{Z}_{\mathrm{b} 4}
\end{aligned}
$$

Rewriting them in matrix form, that is

$$
\boldsymbol{Z}_{\mathrm{T} l}=\boldsymbol{B}_{\mathrm{t} K_{\mathrm{V}}} \boldsymbol{Z}_{\mathrm{bt}} \boldsymbol{B}_{\mathrm{t} K_{\mathrm{I}}}^{\mathrm{T}}+\boldsymbol{Z}_{\mathrm{b} l}
$$

The calculation of $\boldsymbol{B}_{\mathrm{t} h K_{\mathrm{V}}}$ and $\boldsymbol{B}_{\mathrm{t} h K_{\mathrm{I}}}$ for loop $h$ are similar to (7), that is

$$
\begin{aligned}
\boldsymbol{B}_{\mathrm{t} h K_{\mathrm{V}}} & =\boldsymbol{T}_{\mathrm{t} i K_{\mathrm{V}}}-\boldsymbol{T}_{\mathrm{t} j K_{\mathrm{V}}} \\
\boldsymbol{B}_{\mathrm{t} h K_{\mathrm{I}}} & =\boldsymbol{T}_{\mathrm{t} i K_{\mathrm{I}}}-\boldsymbol{T}_{\mathrm{t} j K_{\mathrm{I}}}
\end{aligned}
$$

where, $\boldsymbol{T}_{\mathrm{t} i K_{\mathrm{V}}}$ and $\boldsymbol{T}_{\mathrm{t} j K_{\mathrm{V}}}$ are the node $i$ and node $j$ corresponding row vectors (path vectors) in $\boldsymbol{T}_{\mathrm{t} K_{\mathrm{V}}}, \boldsymbol{T}_{\mathrm{t} i K_{\mathrm{I}}}$ and $\boldsymbol{T}_{\mathrm{t} j K_{\mathrm{I}}}$ are the node $i$ and node $j$ corresponding row vectors (path vectors) in $\boldsymbol{T}_{\mathrm{t} K_{\mathrm{I}}}$.

Then $\boldsymbol{I}_{l}$ can be calculated with (6) when $\boldsymbol{Z}_{\mathrm{T} l}$ and $\Delta \boldsymbol{U}_{l}$ are known. Like (7), the contribution of $\boldsymbol{I}_{l h}$ in $h$ loop to the tree branch currents can be calculated by,

$$
\boldsymbol{f}_{2 h}=\left[\begin{array}{ll}
\boldsymbol{T}_{\mathrm{t} i K_{\mathrm{I}}}^{\mathrm{T}} & \boldsymbol{T}_{\mathrm{t} j K_{\mathrm{I}}}^{\mathrm{T}}
\end{array}\right]\left[\begin{array}{c}
\boldsymbol{I}_{l h} \\
-\boldsymbol{I}_{l h}
\end{array}\right]=\left[\begin{array}{ll}
\boldsymbol{T}_{\mathrm{t} i K_{\mathrm{I}}}^{\mathrm{T}} & -\boldsymbol{T}_{\mathrm{t} j K_{\mathrm{I}}}^{\mathrm{T}}
\end{array}\right] \boldsymbol{I}_{l h}
$$

And then $\boldsymbol{f}_{2}$ can be obtained with (8).

\subsubsection{Modification of the power flow algorithm}

When there are DT branches in the network, the calculation process of power flow is the same as that described in section 2. But it should use the formulas proposed in this section to calculate $\boldsymbol{f}_{1}$ (with(16)), $\Delta \boldsymbol{U}_{l}$ (with(18)), $\boldsymbol{Z}_{\mathrm{T} l}\left(\right.$ with(20)) and $\boldsymbol{f}_{2}($ with(23)).

\section{Compensation for PV Nodes}

When integrating DGs into distribution systems, some DGs can be well represented as PQ nodes, which require little special treatment in the power flow algorithm. Other need be modeled as PV nodes, which inject a given active power into the system and require the necessary but unknown reactive power to sustain the voltage magnitude to a scheduled value. Thus the output reactive powers of PV nodes need to be determined and to compensate PV node voltage mismatch.

The phase injection current of any PV node $i$ can be calculated with (subscript "d" means PV nodes),

$$
\dot{I}_{\mathrm{d} p i}=-\left(\frac{P_{\mathrm{d} i}+\mathrm{j} Q_{\mathrm{d} i}}{3 \dot{U}_{\mathrm{d} p i}}\right)^{*} \quad(p=\mathrm{a}, \mathrm{b}, \mathrm{c} ; i=1,2, \cdots, n)
$$

where, $\dot{U}_{\mathrm{d} p i}$ is the phase voltage phasor with fixed magnitude and its phase angle is unknown, $P_{\mathrm{d} i}$ and $Q_{\mathrm{d} i}$ is the total active power and reactive power (the minus represents that PV node outputs active and reactive powers to the system).

The open loop impedance matrix (Thevenin equivalent impedance matrix) is used to deal with PV node [13-14, 23]. Supposing that there are $N_{\mathrm{d}}$ PV nodes in the system, the relationship between the breakpoint compensation voltage vector $\Delta \boldsymbol{U}_{\mathrm{d}}$ and current vector $\Delta \boldsymbol{I}_{\mathrm{d}}$ is,

$$
\Delta \boldsymbol{U}_{\mathrm{d}}=\boldsymbol{Z}_{\mathrm{d}} \Delta \boldsymbol{I}_{\mathrm{d}}
$$

where $\boldsymbol{Z}_{\mathrm{d}}$ is the Thevenin equivalent impedance matrix of $3 N_{\mathrm{d}} \times 3 N_{\mathrm{d}}$.

For a radial distribution network with DT branches, extracting all PV nodes corresponding row vectors from path matrices $\boldsymbol{T}_{\mathrm{t} K_{\mathrm{V}}}$ and $\boldsymbol{T}_{\mathrm{t} K_{\mathrm{I}}}$ make up two new matrices, $\boldsymbol{T}_{\mathrm{td} K_{\mathrm{V}}}$ and $\boldsymbol{T}_{\mathrm{td} K_{\mathrm{I}}}$, then $\boldsymbol{Z}_{\mathrm{d}}$ can be expressed by [23],

$$
\boldsymbol{Z}_{\mathrm{d}}=\boldsymbol{T}_{\mathrm{td} K_{\mathrm{v}}} \boldsymbol{Z}_{\mathrm{bt}} \boldsymbol{T}_{\mathrm{td} K_{\mathrm{I}}}^{\mathrm{T}}
$$

The sequence impedance matrix for any branch can be expressed as,

$$
\boldsymbol{Z}_{\mathrm{bi} \_012}=\boldsymbol{A}^{-1} \boldsymbol{Z}_{\mathrm{bi}} \boldsymbol{A}
$$

When the spatial distribution of three-phase transmission lines is symmetrical, all non-diagonal elements in $\boldsymbol{Z}_{\mathrm{bi} 012}$ are equal to zero. When the spatial distribution is asymmetrical, non-diagonal element in $\boldsymbol{Z}_{\mathrm{bi} 012}$ is not equal to zero which means that the sequence impedances are coupled. Generally speaking, the absolute values of non-diagonal elements are far less than those of the diagonal elements, so they can be ignored. Then $\boldsymbol{Z}_{\mathrm{bi} 012}$ can be simplified as,

$$
\boldsymbol{Z}_{\mathrm{bi} \_012}=\operatorname{diag}\left(\begin{array}{lll}
\boldsymbol{Z}_{\mathrm{bi} \_0} & \boldsymbol{Z}_{\mathrm{bi} \_} & \boldsymbol{Z}_{\mathrm{bi} \_}
\end{array}\right)
$$


Let $\boldsymbol{A}_{\mathrm{d}}=\operatorname{diag}(\boldsymbol{A}, \cdots, \boldsymbol{A})$ (total of $\left.N_{\mathrm{d}} \boldsymbol{A}\right)$ and premultiplying (25) with $\boldsymbol{A}_{\mathrm{d}}^{-1}$ gives,

$$
\begin{aligned}
\Delta \boldsymbol{U}_{\mathrm{d} \_012} & =\boldsymbol{A}_{\mathrm{d}}^{-1} \Delta \boldsymbol{U}_{\mathrm{d}}=\boldsymbol{A}_{\mathrm{d}}^{-1} \boldsymbol{Z}_{\mathrm{d}} \Delta \boldsymbol{I}_{\mathrm{d}} \\
& =\boldsymbol{A}_{\mathrm{d}}^{-1} \boldsymbol{Z}_{\mathrm{d}} \boldsymbol{A}_{\mathrm{d}}\left(\boldsymbol{A}_{\mathrm{d}}^{-1} \Delta \boldsymbol{I}_{\mathrm{d}}\right)=\boldsymbol{Z}_{\mathrm{d} \_012} \Delta \boldsymbol{I}_{\mathrm{d} \_012}
\end{aligned}
$$

It can be seen from (28) that the sequence impedances of each branch can be approximated as a diagonal matrix, so the positive sequence component equation can be extracted from (29) as,

$$
\Delta \boldsymbol{U}_{\mathrm{d}_{-} 1}=\boldsymbol{Z}_{\mathrm{d}_{-} 1} \Delta \boldsymbol{I}_{\mathrm{d}_{-} 1}
$$

The dimensions of $\Delta \boldsymbol{U}_{\mathrm{d}_{1} 1}, \Delta \boldsymbol{I}_{\mathrm{d}_{-} 1}$ and $\boldsymbol{Z}_{\mathrm{d}_{-} 1}$ are equal to the number of PV nodes, $N_{\mathrm{d}}$. Define,

$$
\begin{aligned}
& \Delta \boldsymbol{U}_{\mathrm{d} \_1}=\Delta \boldsymbol{e}_{\mathrm{d}_{-} 1}+\mathrm{j} \Delta \boldsymbol{f}_{\mathrm{d}_{-} 1} \\
& \Delta \boldsymbol{I}_{\mathrm{d}_{-} 1}=\Delta \boldsymbol{c}_{\mathrm{d}_{1} 1}+\mathrm{j} \Delta \boldsymbol{d}_{\mathrm{d}_{-} 1} \\
& \boldsymbol{Z}_{\mathrm{d}_{-} 1}=\Delta \boldsymbol{R}_{\mathrm{d}_{-} 1}+\mathrm{j} \Delta \boldsymbol{X}_{\mathrm{d}_{-} 1}
\end{aligned}
$$

Substituting (31) (33) into (30) and rewriting it,

$$
\left[\begin{array}{cc}
\boldsymbol{X}_{\mathrm{d}_{-} 1} & \boldsymbol{R}_{\mathrm{d}_{-} 1} \\
-\boldsymbol{R}_{\mathrm{d}_{-} 1} & \boldsymbol{X}_{\mathrm{d}_{-} 1}
\end{array}\right]\left[\begin{array}{c}
-\Delta \boldsymbol{d}_{\mathrm{d} \_1} \\
\Delta \boldsymbol{c}_{\mathrm{d}_{-} 1}
\end{array}\right]=\left[\begin{array}{c}
\Delta \boldsymbol{e}_{\mathrm{d}_{-} 1} \\
\Delta \boldsymbol{f}_{\mathrm{d}_{-} 1}
\end{array}\right]
$$

Let $U_{\mathrm{dri}}$ be the scheduled voltage magnitude for PV node $i$. During the $k$ th iteration, the positive sequence voltage $\dot{U}_{\mathrm{d} i}^{k}{ }_{1}$ of breakpoint is calculated with phase angle $\theta_{\mathrm{d} i_{1} 1}^{k}$ (both sides of the breakpoint should have the same phase angle), then voltage mismatch for PV node $i$ can be calculated by,

$$
\Delta \dot{U}_{\mathrm{d} i_{-} 1}^{k}=\left(U_{\mathrm{dr} i}-U_{\mathrm{d} i_{-} 1}^{k}\right) \mathrm{e}^{\mathrm{j} \theta_{\mathrm{d}{ }_{-1}}^{k}}=\Delta e_{\mathrm{d} i_{-} 1}^{k}+\mathrm{j} \Delta f_{\mathrm{d} i_{-} 1}^{k}
$$

The reactive power increment at PV node $i$ will be determined by,

$$
\Delta Q_{\mathrm{d} i}^{k+1}=-\frac{3 U_{\mathrm{dri}} \Delta d_{\mathrm{d} i_{-} 1}^{k}}{\cos \theta_{\mathrm{d}{ }_{-} 1}}
$$

The PV node reactive current injection $\Delta d_{\mathrm{d} i-1}^{k}$ will be solved using following equation,

$$
\boldsymbol{X}_{\mathrm{d}_{-} 1}\left(-\Delta \boldsymbol{d}_{\mathrm{d} i_{-} 1}^{k}\right)=\Delta \boldsymbol{e}_{\mathrm{d} i_{-} 1}^{k}
$$

where, $\Delta \boldsymbol{e}_{\mathrm{d} i_{1} 1}^{k}$ can be obtained from (34). Appendix B describes the derivation of (36) and (37) in detail.

The total reactive power at $\mathrm{PV}$ node $i$ is,

$$
Q_{\mathrm{d} i}^{k+1}=Q_{\mathrm{d} i_{-} 1}^{k}+\Delta Q_{\mathrm{d} i}^{k+1}
$$

The initial value of output reactive power could be set to 0 or other value. And $Q_{\mathrm{d} i}^{k+1}$ is compared with the reactive power generation limits at PV node $i$. If $Q_{\mathrm{d} i}^{k+1}$ is within the limits, the corresponding current injection is calculated according to (24). Otherwise, if $Q_{\mathrm{d} i}^{k+1}$ violates any reactive power limit, it will be set to that limit and PV node $i$ will be converted to $P Q$ node during next iteration, and in addition, the row and column in $\boldsymbol{X}_{\mathrm{d}_{-} 1}$, corresponding to this node, are removed.

\section{Iterative Process of Hybrid Power Flow Algorithm}

The overall power flow algorithm should consist of two nested iteration loops for meshed power flow calculation (inner loop) and PV node voltage magnitude compensation calculation (outer loop) respectively. The inner loop calculates the meshed power flow by regarding PV nodes as PQ nodes, and the termination is controlled by the maximum node voltage mismatch between the results of two successive iterations. The outer loop calculate the reactive power increments at PV nodes with (36) and then reenter the inner loop until the maximum voltage mismatch ate each PV node is small enough.

However, the experience shows that the current injection increments generated by the reactive power increments from PV nodes have little impact on the power flow of the whole system at each iteration. So the outer loop can be cancelled for the output reactive powers at PV nodes can be updated at each iteration during the inner loop. A simple flow chart of overall power flow algorithm is shown in Fig.4. During each iteration, the meshed power flow is calculated first, then the reactive power increments are calculated, and the next iteration starts. Hence, the higher calculation efficiency can be obtained.

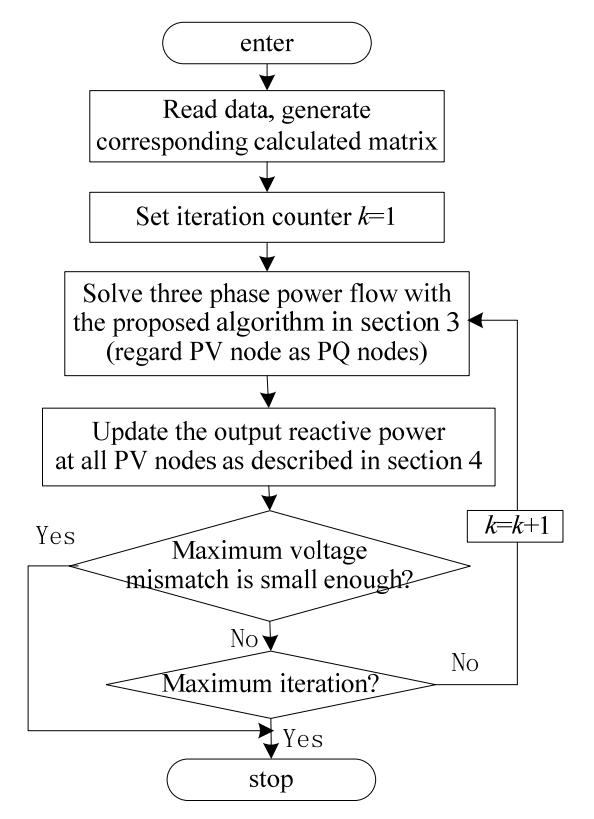

Fig. 4. A simple flow chart of the hybrid algorithm 


\section{Numerical Results}

\subsection{Test 1: IEEE 4 node test feeder}

IEEE four-node test feeder [25] was used to validate the proposed algorithm and DT models. The test results of the algorithm match very well with those listed in [25] for both balanced and unbalanced loads with different DT connections (the convergence accuracy is $10-6$ p.u.). The number of iterations is six for all cases. The feasibility of the proposed method and the correctness of DT models have been proved.

\subsection{Test 2: modified IEEE 34 node test feeder}

Another test feeder is IEEE 34 test feeder [25] but all the regulators are excluded from the standard data. The test feeder was characterized for its very long lines and light loads. The system maximum load is $1165 \mathrm{~kW}$ and 665 kvar with unbalanced distribution. Because of the length of the feeder and the unbalanced loads, it might have a convergence problem.

The feeder has one $\Delta-Y n$ DT, T1, at substation and another one, T2, on the lateral near node 13. For testing purposes, four loops were added and two more DTs, T3 and T4, were introduced. The one-line diagram of the modified 34 feeder is shown in Fig. 5. Similar to T2, both T3 and T4 reduce the voltage from 24.9 to $4.16 \mathrm{kV}$. There's a fixed capacity parallel capacitor group at node 31 and the reactive compensation capacity is 300 kvar.

Let T2 T4 have the same winding connections. The converged iterations with the proposed power flow method for different connections are summarized in Table 2 (convergence accuracy is $10^{-6}$ p.u., voltage) .

Radial Distribution Analysis Package (RDAP) [26] was further used to verify the correctness of the proposed

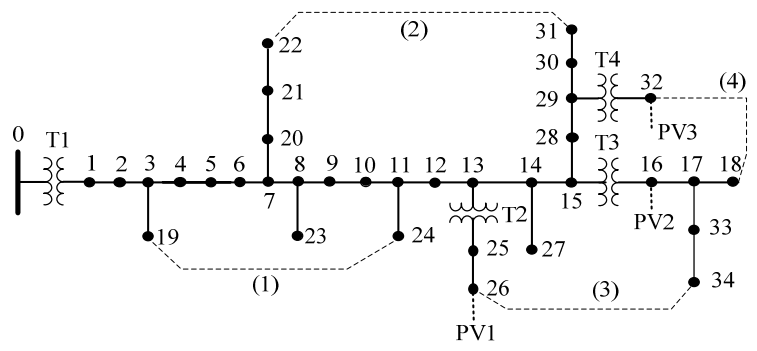

Fig. 5. The modified IEEE 34 node test feeder

Table 2. Converged results of Test 2

\begin{tabular}{c|c|c|c|c|c|c}
\hline \multirow{2}{*}{ Item } & \multirow{2}{*}{$\begin{array}{c}\mathrm{T} 2 \sim \mathrm{T} 4 \\
\text { connection }\end{array}$} & \multicolumn{6}{|c}{ closed loops } \\
\cline { 3 - 7 } & \multirow{3}{*}{$\mathrm{Y}_{\mathrm{n}}-\mathrm{Y}_{\mathrm{n}}$} & 11 & 11 & 12 & 12 & 12 \\
\cline { 3 - 7 } & & 6 & 6 & 7 & 7 & 7 \\
\cline { 3 - 7 } & & 11 & 10 & 10 & 9 & 9 \\
\cline { 3 - 7 } & $\mathrm{Y}_{\mathrm{n}-\Delta}$ & 8 & 8 & 8 & 7 & 7 \\
\cline { 2 - 7 } & $\Delta-\Delta$ & 9 & 9 & 9 & 8 & 8 \\
\cline { 2 - 7 } & $\Delta-\mathrm{Y}_{\mathrm{n}}$ & 8 & 8 & 8 & 8 & 8 \\
\hline
\end{tabular}

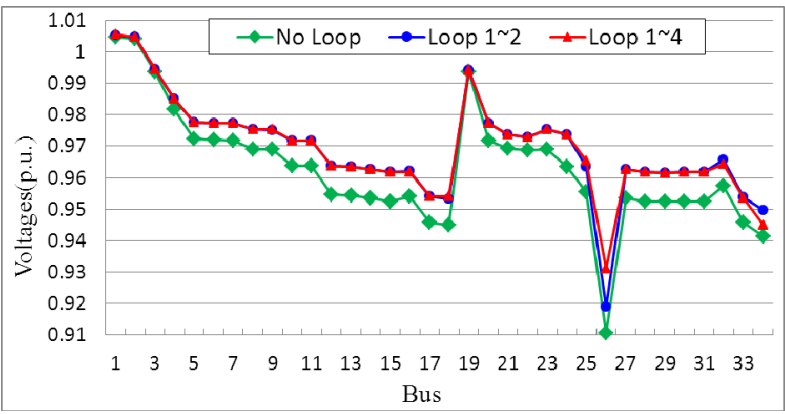

Fig. 6. The A-phase voltage profiles for $Y_{n}-\Delta$ connection of $\mathrm{T} 2 \sim \mathrm{T} 4$

method. The results calculate with the proposed method are found to be in consistency with the RDAP's results for all the connections shown in Table 2.

In order to further examine the algorithm's robustness and accuracy, the method in [6] and the Newton-Raphson method in [3] are used to compare with the proposed methods with $Y_{n}-Y_{n}$ connection of T2 4. The results from three methods are basically consistent and the maximum relative error is below $1 \%$. In Table 2, the underlined numbers (first row for $\mathrm{Y}_{\mathrm{n}}-\mathrm{Y}_{\mathrm{n}}$ ) are the converged iterations with the method in [6], and the italic and underlined numbers (second row for $\mathrm{Y}_{\mathrm{n}}-\mathrm{Y}_{\mathrm{n}}$ ) in Table 2 are the converged iterations with the Newton-Raphson (N-R) method in [3]. The N-R method has better convergence (fewest iterations), but it has much longer calculation time.

Moreover, as shown in Table 2, the proposed method has less iteration number with more loops for meshed network. So compared with those two methods in [6] and [3], the proposed method has the fastest calculation speed and efficiency.

When T2 4 with $\mathrm{Y}_{\mathrm{n}}-\Delta, \Delta-\Delta$ and $\Delta-\mathrm{Y}_{\mathrm{n}}$ connections, the proposed algorithm has less iterations. The possible reason may be that the voltage unbalance degrees for $Y_{n}-Y_{n}$ connection are much larger than those for later three cases.

Fig. 6 shows the A-phase converged voltage profiles for no closed loop, closed loop 1 2 and closed loop1 4 respectively for $\mathrm{Y}_{\mathrm{n}}-\Delta$ connection of T2 T4. It can be seen from Fig. 6 that the closed loops can make better power flows and improve node voltage level although the output active and reactive powers keep the same. The results also indicate that the more closed loops, the better the voltage level.

\subsection{Test 3: modified IEEE 34 node test feeder with PV nodes}

Even with the light loads, the voltages at some nodes are low and do not meet the standards for the larger voltage drops in the long transmission lines. So, three DGs with PV node type are added at node 26,16 and 32 respectively (see Fig. 5). Their rated active powers are $150 \mathrm{~kW}, 100 \mathrm{~kW}$ and $100 \mathrm{~kW}$ in turn. The lower and upper limits of each DG output reactive power are set to zero and the value of the 
Table 3. Converged results of Test 3 with PV nodes

\begin{tabular}{|c|c|c|c|c|c|c|c|c|c|c|}
\hline \multirow{3}{*}{ item } & \multirow{3}{*}{$\begin{array}{c}\mathrm{T} 2 \sim \mathrm{T} 4 \\
\text { Connection }\end{array}$} & \multicolumn{9}{|c|}{ closed loops and PV nodes } \\
\hline & & \multicolumn{3}{|c|}{ no Loop } & \multicolumn{3}{|c|}{ loop $1 \sim 2$} & \multicolumn{3}{|c|}{ loop1 4 } \\
\hline & & PV1 & PV1 2 & PV1 3 & PV1 & PV1 2 & PV1 3 & PV1 & PV1 2 & PV1 3 \\
\hline \multirow{4}{*}{ iterations } & $Y_{n}-Y_{n}$ & 9 & 9 & 9 & 9 & 9 & 9 & 9 & 9 & 9 \\
\hline & $\mathrm{Y}_{\mathrm{n}}-\Delta$ & 7 & 8 & 8 & 7 & 7 & 8 & 7 & 7 & 8 \\
\hline & $\Delta-\Delta$ & 8 & 8 & 8 & 8 & 8 & 8 & 8 & 7 & 8 \\
\hline & $\Delta-Y_{n}$ & 8 & 8 & 8 & 8 & 8 & 8 & 7 & 8 & 11 \\
\hline
\end{tabular}

Table 4. Part of converged results for $\mathrm{Y}_{\mathrm{g}}-\Delta$ connection of T2 T4 in case1 3

\begin{tabular}{|c|c|c|c|c|c|}
\hline item & case & iterations & PV node & positive sequence voltage at PV node/p.u. & reactive power at PV node/kvar \\
\hline \multirow{6}{*}{$\begin{array}{l}\text { Limited reactive } \\
\text { power }\end{array}$} & \multirow{3}{*}{ Case1 } & \multirow{3}{*}{8} & PV1 & 0.99045 & 150 \\
\hline & & & PV2 & 0.99226 & 100 \\
\hline & & & PV3 & 0.99700 & 14.8 \\
\hline & \multirow{2}{*}{ Case 2} & \multirow{2}{*}{8} & PV1 & 0.989651 & 150 \\
\hline & & & PV2 & 0.991288 & 100 \\
\hline & Case3 & 7 & PV1 & 0.96923 & 150 \\
\hline \multirow{6}{*}{$\begin{array}{c}\text { Unlimited reactive } \\
\text { power }\end{array}$} & \multirow{3}{*}{ Case 1} & \multirow{3}{*}{11} & PV1 & 0.99424 & 171.0 \\
\hline & & & PV2 & 0.99430 & 107.9 \\
\hline & & & PV3 & 0.99757 & 0 \\
\hline & \multirow{2}{*}{ Case2 } & \multirow{2}{*}{9} & PV1 & 0.99313 & 154.4 \\
\hline & & & PV2 & 0.99572 & 122.6 \\
\hline & Case3 & 8 & PV1 & 0.99442 & 302.3 \\
\hline
\end{tabular}

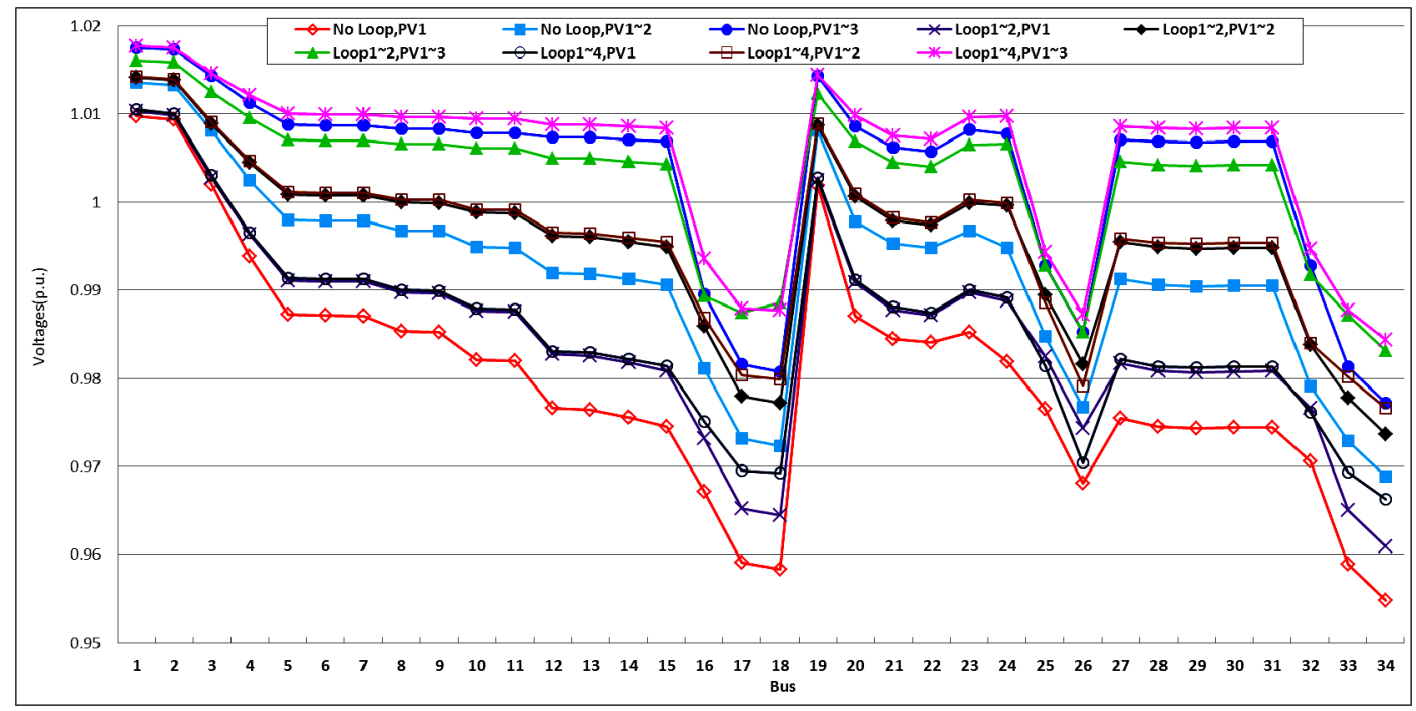

Fig. 7. The A-phase voltage profiles for $\mathrm{Y}_{\mathrm{n}}-\Delta$ connection of T2 T4 with different loops and PV nodes

rated active power respectively. The convergence accuracy is $10^{-6} \mathrm{p} . \mathrm{u}$ (voltage) and the $\mathrm{PV}$ voltage mismatch is 0.01 p.u. The converged iterations for different operation modes with the proposed method are shown in Table 3.

Comparing the results in Table 2 and Table 3, it can be seen that the iteration numbers changed little with PV nodes, so the good convergence of the proposed algorithm is further verified. The A-phase converged voltage profiles for $Y_{n}-\Delta$ connection of T2 T4 with different loops and PV nodes are shown in Fig. 7. It can be seen that the voltages have been improved with closed loops and (or) PV nodes. Similarly, the more closed loops and (or) PV nodes, the better the improvement of the voltage level.
Some PV nodes will be converted to PQ node if they violate the reactive power limits. Generally speaking, the voltage can be further improved by increasing the output reactive power. In order to further analyze the effect on the voltage level from the output reactive power of PV node, three cases were discussed for $\mathrm{Y}_{\mathrm{n}}-\Delta$ connection of T2 T4,

Case1: no closed loop but including PV1 3;

Case2: closed loop 1 2 and PV1 2;

Case3: closed loop 1 4 and PV1;

The Fig. 8 is the A-phase voltage profiles for $\mathrm{Y}_{\mathrm{n}}-\Delta$ connection of T2 T4 for limited and unlimited output reactive power at each PV node for three cases. And the 


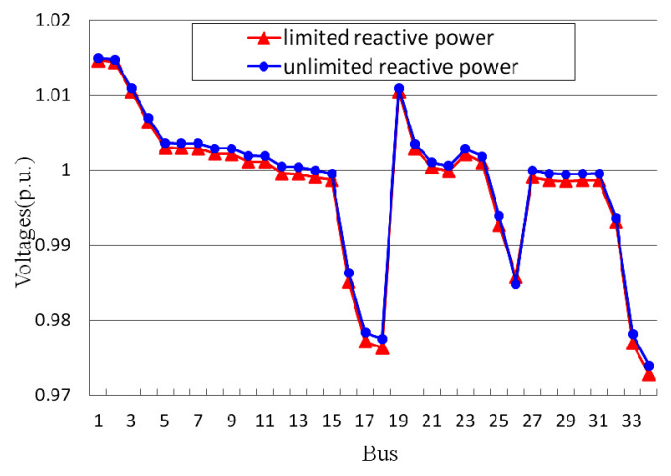

(a) Case 1

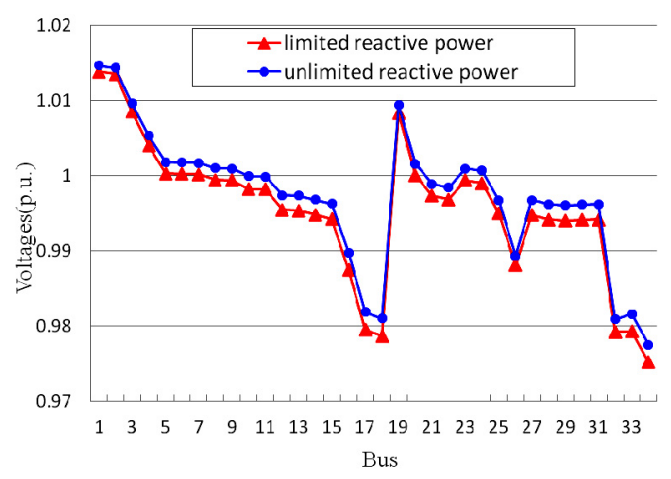

(b) Case2

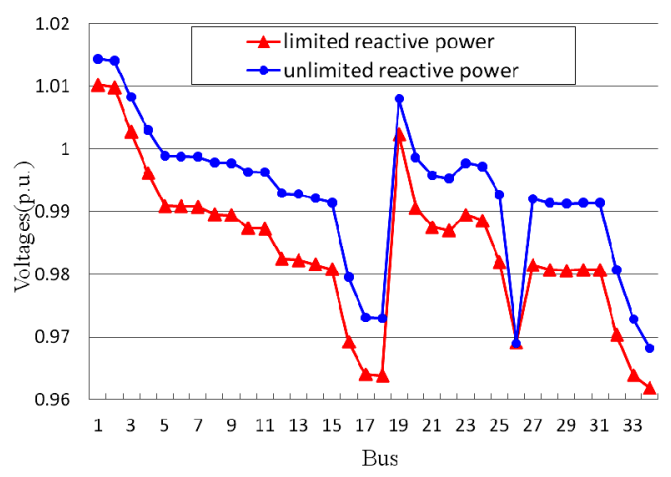

(c) Case 3

Fig.8. The A-phase voltage profiles for $Y_{n}-\Delta$ connection of T2 T4 for limited and unlimited reactive power at PV node

related final solutions are presented in Table 4.

In three cases, the voltage level with the unlimited output reactive power is better than that with limited output reactive power The voltage improvement in case 3 is more obvious (see Fig. 8(c)), but the iteration number increases. From Table 4 for case 3, when the output reactive power has a limit, the positive sequence voltage at the PV node (PV1) is very low, 0.96923 p.u.. When the output reactive power has no limit, it is up to 0.99442 p.u.. However, this has been achieved with the cost of more reactive power (the reactive power has doubled from 150kvar to $302.3 \mathrm{kvar}$ in Case 3), and there will be much difficult in the actual system due to the high investments.

\section{Conclusion}

This paper presents a three-phase hybrid power flow solution for meshed distribution systems with DT branches and PV nodes. The main works include,

An improved three-phase power flow solution for meshed distribution system was presented based on node-branch incidence matrix. The method has clear theory basis and good efficiency.

The detailed DT models for all different connections were re-developed by considering the DT impedance asymmetry and neutral point grounded impedance. The equivalent model of DT branch was established and can be dealt with as an ordinary transmission line with the proposed power flow method.

Based on the assumption that the positive-sequence voltage magnitudes of PV nodes are invariable, an approach was developed to calculate reactive power increments of $\mathrm{PV}$ nodes. It can be easily integrated into the propose power flow method.

Compared with the typical methods by using the IEEE4 and the modified IEEE34 test feeders, the validity and efficiency of the proposed algorithm have been proved.

\section{Acknowledgements}

This work is supported financially by the Chinese National Key Basic Research and Development Program (973) with item \#2013CB228203 and the Chinese Postdoctoral Science Foundation with item \#2014M562335.

\section{References}

[1] T. Chen, M. Chen, K. Hwang, P. Kotas, and E. Chebli, "Distribution system power flow analysis: A rigid approach," IEEE Trans. Power Deliv., vol. 6, no. 3, pp. 1146-1152, 1991.

[2] Ray D. Zimmerman, Hsiao-Dong Chiang, "Fast decoupled power flow for unbalanced radial distribution systems," IEEE Trans. Power Syst., vol. 10, no. 4, pp. 2045-2051, 1995.

[3] Zhang F, Cheng C S, "A Modified Newton Method for Radial Distribution System Power Flow Analysis," IEEE Trans. Power Syst., vol. 12, no. 1, pp. 389-397, 1997.

[4] P. A. N. Garcia, J. L. R. Pereira, S. Carneiro, et al, "Three-phase power flow calculations using the current injection method," IEEE Trans. Power Syst., vol.15, no. 2, pp. 508-514, 2000.

[5] M. Abdel-Akher, K. Nor, and A. Rashid, "Improved three-phase power-flow methods using sequence components," IEEE Trans. Power Syst., vol. 20, no. 3, pp. 1389-1397, 2005. 
[6] Shirmohammadi D, Hong H W, Semlyen A, et al, "A compensation-based power flow method for weakly meshed distribution and transmission networks," IEEE Trans. on Power Syst., vol. 3, no. 2, pp. 753-761, 1988.

[7] Luo G X, Semlyen A, "Efficient load flow for large weakly meshed networks," IEEE Trans. on Power Syst., vol. 5, no. 4, pp. 1309-1316, 1990.

[8] C. Cheng and D. Shirmohammadi, "A three-phase power flow method for real-time distribution system analysis," IEEE Trans. Power Syst., vol. 10, no. 2, pp. 671-679, May 1995.

[9] Teng J H, “A direct approach for distribution system load flow solutions," IEEE Trans. on Power Deliv., vol.18, no.3, pp. 882-887, 2003.

[10] W.C. Wu , B.M. Zhang, "A three-phase power flow algorithm for distribution system power flow based on loop-analysis method," Int. J. Elect. Power and Energy Syst., vol. 30, no. 1, pp. 8-15, 2008.

[11] Augugliaro, L. Dusonchet, S. Favuzza, et al, "A backward sweep method for power flow solution in distribution networks," Int. J. Elect. Power and Energy Syst., vol. 32, no. 1, pp. 271-280, 2010.

[12] T. H. Chen, M. S. Chen, T. Inoue, P. Kotas, and E. A. Chebli, "Three phase cogenerator and transformer models for distribution system analysis," IEEE Trans. Power Deliv., vol.6, no.4, pp1671-1681, 1991.

[13] Mesut E. Baran, Eric A. Staton, "Distribution Transformer Models for Branch Current Based Feeder Analysis," IEEE Trans. on Power Syst., vol. 12, no. 2, pp. 698-703, 1997.

[14] Chen T. H., Yang W. C., Guo T. Y., and Pu G. C., "Modeling and analysis of asymmetrical three-phase distribution transformer banks with mid-tap connected to the secondary neutral conductor," Elect. Power Syst. Research, vol. 54, no. 2, pp. 83-89, 2000.

[15] Irving M. R. and Al-Othman A. K., "Admittance matrix models of three-phase transformers with various neutral grounding configurations," IEEE Trans. Power Syst., vol. 18, no. 3, pp. 1210-1212, Aug. 2003.

[16] Xiao P., Yu D.C., and Yan W., “A Unified Three-Phase Transformer Model for Distribution Load Flow Calculations," IEEE Trans. Power Syst., vol. 21, no. 3, pp. 153-159, 2006.

[17] Wang Z., Chen F., and Li J., "Implementing transformer nodal admittance matrices into backward / forward sweep-based power flow analysis for unbalanced radial distribution systems," IEEE Trans. Power Syst., vol. 19, no. 4, pp. 1831-1836, 2004.

[18] LI hong-wei, LIU Qing-you, SHEN Xia, “A ThreePhase Unbalanced Power Flow Solution with Threephase Distribution Transformers," in Proceedings of DRPT2011, Weihai, China, pp. 831-836, July, 2011.

[19] R. Palma-Behnke, J.L.A. Cerda, L.S. Vargas, A. Jofre, "A distribution company energy acquisition market model with integration of distributed generation and load curtailment options," IEEE Trans. Power Syst., vol. 20, no. 4, pp. 1718-1727, 2005.

[20] Pecas Lopes J A, Hatziargyriou N, Mutale J, et al, "Integrating distributed generation into electric power systems: a review of drivers, challenges and opportunities," Elect. Power Syst. Research, vol.77, no. 9, pp. 1189-1203, 2007.

[21] Y. Zhu and K. Tomsovic, "Adaptive power flow method for distribution systems with dispersed generation," IEEE Trans. Power Deliv., vol. 17, no. 7, pp. 822-827, 2002.

[22] Ding M., Guo X.F. , "Three-phase power flow for the weakly meshed distribution network with the distributed generation," Proceedings of the CSEE, vol. 29, no. 13, pp. 35-40, 2009.

[23] Li H.W., Sun H.B., Zhang A, et al, "Positive-sequence component based three-phase unbalanced power flow solution for distribution system with PV nodes," Proceedings of the CSEE, vol. 32, no. 1, pp. 115-121, 2012.

[24] Hongwei Li, Anan Zhang, Xia Shen, Jin Xu, "A load flow method for weakly meshed distribution networks using powers as flow variables," Int. J. Elect. Power and Energy Syst., vol. 58, pp. 291-299, 2014

[25] Distribution System Analysis Subcommittee. Radial distribution test feeders [EB/OL]. http://ewh.ieee.org /soc/pes/dsacom/testfeeders/ index.html.

[26] William H. Kersting, Distribution System Modeling and Analysis. 2nd. New York: Taylor \& Francis, 2007.

\section{Appendix A}

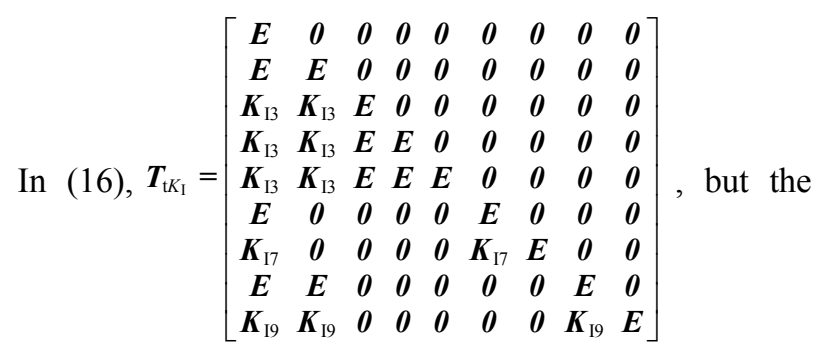

$\left[\begin{array}{lllllllll}E & 0 & 0 & 0 & 0 & 0 & 0 & 0 & 0\end{array}\right.$

$\begin{array}{lllllllll}E & E & 0 & 0 & 0 & 0 & 0 & 0 & 0\end{array}$

$\begin{array}{lllllllll}E & E & E & 0 & 0 & 0 & 0 & 0 & 0\end{array}$

$\begin{array}{llllllllll}E & E & E & E & 0 & 0 & 0 & 0 & 0\end{array}$

path matrix is $\quad \boldsymbol{T}_{\mathrm{t}}=\begin{array}{lllllllll}\boldsymbol{E} & \boldsymbol{E} & \boldsymbol{E} & \boldsymbol{E} & \boldsymbol{E} & \boldsymbol{0} & \boldsymbol{0} & \boldsymbol{0} & \boldsymbol{0}\end{array}$ when

$\begin{array}{lllllllll}E & 0 & 0 & 0 & 0 & E & 0 & 0 & 0\end{array}$

$\begin{array}{lllllllll}E & 0 & 0 & 0 & 0 & E & E & 0 & 0\end{array}$

$\begin{array}{llllllllll}E & E & 0 & 0 & 0 & 0 & 0 & E & 0\end{array}$

$\left[\begin{array}{lllllllll}E & E & 0 & 0 & 0 & 0 & 0 & E & E\end{array}\right]$

replacing all DT branches with ordinary transmission lines in Fig. 3. Comparing $\boldsymbol{T}_{\mathrm{t}}$ with $\boldsymbol{T}_{\mathrm{t} K_{\mathrm{I}}}$, the generation process of $\boldsymbol{T}_{\mathrm{t} K_{\mathrm{I}}}$ can be summarized as following.

Moving from the first entry to the last entry along the path of node $i$ in $\boldsymbol{T}_{\mathrm{t}}$, when encountering DT branch $j$, the corresponding entries before DT branch $j$ corresponding entry are pre-multiplied with $\boldsymbol{K}_{\mathrm{I} j}$ and then path vector $\boldsymbol{T}_{\mathrm{t} i K_{\mathrm{V}}}$ of node $i$ could be obtained. Certainly, if there is more 
than one DT branch on the path, all the corresponding entries should be pre-multiplied with corresponding $\boldsymbol{K}_{\mathrm{I}}$ whenever encountering a DT branch along the path.

In Fig. 3 the voltages of node 8 and node 9 can be calculated with:

$$
\begin{aligned}
& \boldsymbol{U}_{\mathrm{n} 8}=\boldsymbol{U}_{0}-\boldsymbol{Z}_{\mathrm{b} 1} \boldsymbol{I}_{\mathrm{bt} 1}-\boldsymbol{Z}_{\mathrm{b} 2} \boldsymbol{I}_{\mathrm{bt} 2}-\boldsymbol{Z}_{\mathrm{b} 8} \boldsymbol{I}_{\mathrm{bt} 8} \\
& =\boldsymbol{U}_{0}-\left[\begin{array}{lllllllll}
\boldsymbol{E} & \boldsymbol{E} & \boldsymbol{0} & \boldsymbol{0} & \boldsymbol{0} & \boldsymbol{0} & \boldsymbol{0} & \boldsymbol{E} & \boldsymbol{0}
\end{array}\right] \boldsymbol{Z}_{\mathrm{bt}} \boldsymbol{I}_{\mathrm{bt}} \\
& =\boldsymbol{U}_{0}-\boldsymbol{T}_{\mathrm{t} 8 K_{\mathrm{V}}} \boldsymbol{Z}_{\mathrm{bt}} \boldsymbol{I}_{\mathrm{bt}} \\
& \boldsymbol{U}_{\mathrm{n} 9}=\boldsymbol{K}_{\mathrm{v} 9}\left(\boldsymbol{U}_{0}-\boldsymbol{Z}_{\mathrm{b} 1} \boldsymbol{I}_{\mathrm{bt} 1}-\boldsymbol{Z}_{\mathrm{b} 2} \boldsymbol{I}_{\mathrm{b} 12}-\boldsymbol{Z}_{\mathrm{b} 8} \boldsymbol{I}_{\mathrm{bt} 8}\right)-\boldsymbol{Z}_{\mathrm{T} 9} \boldsymbol{I}_{\mathrm{bt} 9} \\
& =\boldsymbol{K}_{\mathrm{v} 9} \boldsymbol{U}_{0}-\left[\begin{array}{llllllllll}
\boldsymbol{K}_{\mathrm{v} 9} & \boldsymbol{K}_{\mathrm{v} 9} & \boldsymbol{O} & 0 & 0 & 0 & 0 & \boldsymbol{K}_{\mathrm{v} 9} & \boldsymbol{E}
\end{array}\right] \boldsymbol{Z}_{\mathrm{bt}} \boldsymbol{I}_{\mathrm{bt}} \\
& =\boldsymbol{K}_{\mathrm{v} 9} \boldsymbol{U}_{0}-\boldsymbol{T}_{\mathrm{t} 9 K_{\mathrm{v}}} \boldsymbol{Z}_{\mathrm{bt}} \boldsymbol{I}_{\mathrm{bt}}
\end{aligned}
$$

where, $\boldsymbol{Z}_{\mathrm{T} 9}$ is determined by DT coefficient matrices, $\boldsymbol{Z}_{\mathrm{bt}}$ is link branches impedance diagonal matrix of $3 m \times 3 m, \quad \mathrm{~T}_{\mathrm{t} 8 K_{\mathrm{V}}}$ and $\mathrm{T}_{\mathrm{t} 9 K_{\mathrm{V}}}$ are the node 8 and node 9 corresponding row vectors in $\mathrm{T}_{\mathrm{t} K_{\mathrm{V}}}$ respectively.

By introducing the voltage calculation process to other nodes based on (A.1) and (A.2), the formula to calculate node voltage can be expressed in general form as (17).

\section{Appendix B}

After $k$ th iteration, the positive sequence current increment at PV node $\mathrm{i}$ is $\Delta I_{\mathrm{d} i_{-}}^{k}$, and then the apparent power increment at PV node i can be calculated by,

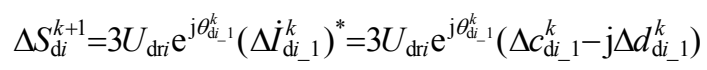

Expanding (B.1), the active power increment is,

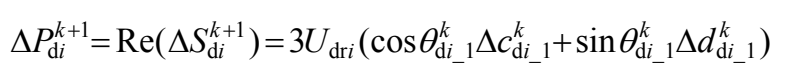

For the output active power of PV node is constant, that is $\Delta P_{\mathrm{d} i}^{k+1}=0$. Substituting it into (B.2) gives

$$
\Delta c_{\mathrm{d} i_{-} 1}^{k}=-\Delta d_{\mathrm{d} i_{-} 1}^{k} \tan \theta_{\mathrm{d} i_{-} 1}^{k}
$$

From (B.1), the reactive power increment of PV node $i$ can be expressed as,

$$
\Delta Q_{\mathrm{d} i}^{k+1}=3 U_{\mathrm{dri}}\left(\sin \theta_{\mathrm{d} i_{-} 1}^{k} \Delta_{\mathrm{d} i_{-1}-\cos }^{k} \theta_{\mathrm{d} i_{-} 1}^{k} \Delta d_{\mathrm{d}_{-} i_{1}}^{k}\right)
$$

Substituting (B.3) into (B.4) and rearranging gives (36).

Besides, $\theta_{\mathrm{d} i_{-} l_{k}}$ is generally very small, so $\left|\Delta \boldsymbol{c}_{\mathrm{d}_{1}}\right|$ is much less than $\left|\Delta d_{\mathrm{d}_{1} 1}\right|$ based on (B.3). Then (37) can be obtained by expanding (34) and ignoring $\Delta \boldsymbol{c}_{\mathrm{d} \_1}$.

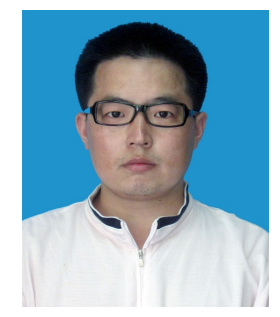

Hongwei Li He received Ms.c degree in electrical engineering from Southwest Jiaotong University in China in 2005. His research interests are Distribution system analysis and control, Distributed Generation modeling and Control.

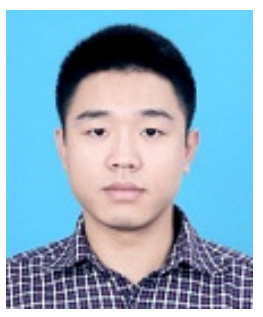

Huabing Wu He received B.S degree in Electrical Engineering and Automation from Southwest Petroleum University in 2013. Now, he is a graduate student in Control Science and Engineering in Southwest Petroleum University. His research interests are microgrid analysis and optimization.

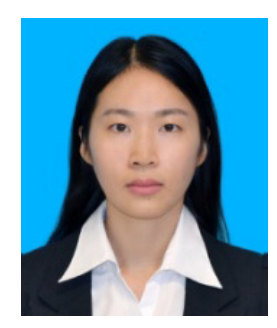

Biyu Jiang She received B.S degree in electrical engineering and automation from southwest petroleum university in 2013. Now she is a graduate student in control science and engineering in Southwest Petroleum University. Her research interest is microgrid analysis and optimization.

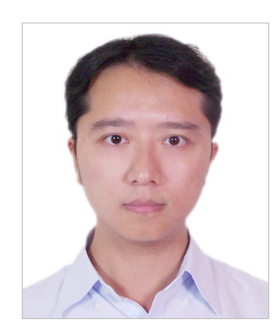

Anan Zhang He received B.S., M.S. and $\mathrm{PhD}$ degree from Sichuan University in 2000, 2003 and 2010 respectively. His research interests are smart grid, reactive power control and voltage control.

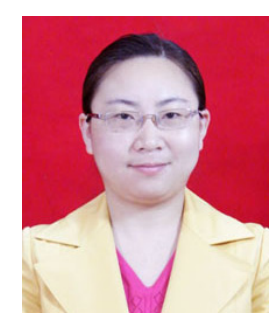

Wei Fang She received B.S. degree in testing technology and automatic instrument from Chongqing University in 1998. Her research interests are power electronics and power drives. 